\title{
The Application of WebQuest in English Teaching
}

\author{
Jia Suo
}

Department of Foreign Languages, North China Electric Power University, Baoding, Hebei, China (suojiasj@ 163.com)

Abstract - There are great similarities between WebQuest and the output-driven hypothesis in the following aspects: the theme, the objective, the procedure and the evaluation. Therefore they can be integrated in a perfect way. WebQuest offers a convenient platform for the implement of the output-driven hypothesis.

Keywords-WebQuest, the output-driven hypothesis, similarities, integration

\section{WebQuest 在英语教学中的应用}

\author{
索佳 \\ 华北电力大学英语系, 保定, 河北, 中国
}

\begin{abstract}
摘 要 WebQuest 与“输出驱动假设”的学习主题类似, 学习的宗旨及目标相同, 实施流程相似, 评价体系一致, 因此两者可以进 行完美结合, WebQuest 为“输出驱动假设”的实施提供了一个方便有效的平台。

关键词 WebQuest, “输出驱动假设”, 相似点, 结合
\end{abstract}

\section{1. 引言}

现代教育技术已经对英语教学起到了非常重要的辅助 作用, 比如多媒体技术在英语教学中的应用提高了学生的 学习兴趣, 改善了授课效果。目前风靡世界的 WebQuest 也将为英语教学带来天翻地覆的变化。

\section{WebQuest}

\section{1 概述}

WebQuest 是网络环境下的主题探究学习活动, 是用于 实现“研究性学习”教学模式的一种教学设计流程模板。它 具有以下四个方面的特征: 第一, WebQuest 的主题是一个 需要解决的问题或者一个需要完成的项目, 即现实生活中 的真实任务。第二, 在 WebQuest 这种活动中, 学生使用的 全部或大部分信息都是从网上获得的。第三, 由于 WebQuest 为教师提供有固定结构的教学设计流程模板和 一系列的指导信息，这就相当于为一线教师提供了一种便 于掌握、运用教学设计新理念的脚手架, 从而使广大教师

本项目受“中央高校基本科研业务费专项资金” 和 “华北电力大 学本科教育教学改革研究项目”资助。( 资助号: 2014 MS180)
易于上手、易于实施。第四, WebQuest 打破了传统单一的 总结性评价方式, 建立了包括诊断性评价、形成性评价和 总结性评价的完善的评价体系。

\section{2 实施流程}

Bemie Dodge 认为 WebQuest 模式一般应由七个要素或 环节组成: “设计一个合适的课程单元”(简称“引言”)、“选 择一个能促进高级认知发展的任务”(简称“任务”)、“开始网 页设计”、“形成评价”、“制定学习活动过程”、“以文字形式 记下所有活动内容以供别人借鉴”、“检查并改进”。除此以 外, 在多年实际推广应用的过程中, 还形成了其他一些 WebQuest 模式。例如, 包含引言、任务、过程、资源、评 价、总结等六个要素或环节的 WebQuest 模式, 以及包含引 言、任务、过程、评价、结论等五个要素或环节的 WebQuest 模式。“引言”部分的目的主要有两个方面: 一是给学习者 指定方向；二是通过各种手段提升学习者的兴趣。“任务” 模块是对练习结束时学习者对要完成的项目进行描述。在 “过程”模块中, WebQuest 提供了一个“脚手架”引导学生经 历体验专家的思维过程。“脚手架”意味着将令人望而生畏 的项目计划打碎成若干个片段, 让学生能够继续钻研相对 
单一的任务, 引导他们通过研究相对困难的步骤, 从而能 够运用他们的知识。教师给出学习者完成任务将要经历的 步骤, 让学习者知道完成任务的过程。“资源”是一个网站 清单, 教师事先已查找过这些网站。资源经过了预选, 以 便学习者能在主题上集中注意力, 而不是漫无目的地网上 冲浪。Webquest 也向学生提供容易采用的离线资源。“评价” 是用来测评学习结果的, 用量规详细说明了教师如何评估 学生的学习活动, 以及学生如何对学习过程进行相互评估 和自我评估。其常用的评价方法有电子学习档案袋法, 问 卷调查法, 作品分析法和课堂观察法。“结论” 部分提供机 会给学习者总结经验, 鼓励对过程的反思, 拓展和概括所 学知识, 鼓励学习者在其他领域拓展其经验。

\section{3. “输出驱动假设”}

\section{1 概述}

学生之所以对英语学习不感兴趣, 其部分原因也在于 他们觉得不能“学以致用”。所以“学生能用所学的东西完成 什么实际任务? ”应该成为衡量教学质量的标准。因此, 文 秋芳, 中国外语教育研究中心主任, 近年来提出了“输出驱 动假设”这一全新的英语教学理念, 即通过完成交际任务促 进语言学习, 提高学生的学习动力, 因为在学生需求的时 候供给, 他们才会珍惜并利用这份供给。“输出驱动假设” 主张教学要以输出为出发点和终极目标, 因为输出比输入 对外语学习的内驱力更大, 输出驱动有助于盘活高中毕业 生在过去英语学习中积累的“惰性知识”, 可以促进接受性 语言知识的运用, 而且可以激发学生学习新语言知识的欲 望。该假设将职场需要的输出能力作为教学考核目标。

\section{2 教学流程与教学方法}

下图展示了实施输出驱动教学的基本流程与教学方 法。

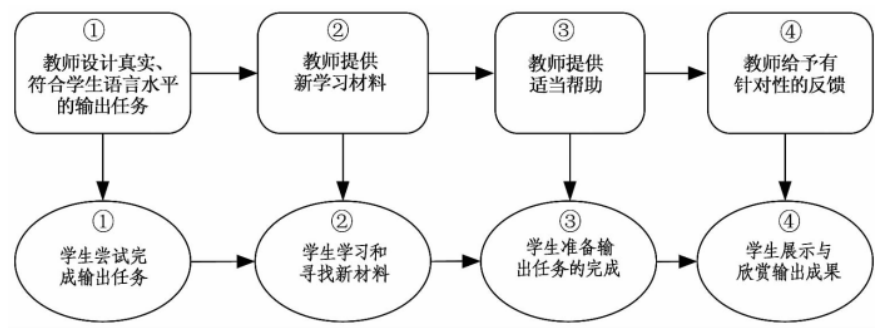

\subsection{1＼cjkstart教师任务}

1) 设计真实、符合学生语言水平的输出任务。根据课 程教学目标, 教师需要在课前设计口头表达、笔头表达、
口译或者笔译任务, 甚至是结合多种产出活动的综合任务。 这些任务需要具有交际真实性, 即所设计的任务是在日常 生活中确实有可能面对的。

2) 提供恰当的新输入材料。其目的是帮助学生补充完 成输出任务所欠缺的语言知识。

3) 提供适当的输出帮助。教师可采取多种形式向学生 提供输出帮助。例如, 与学生讨论输出活动中语言和内容 组织方面的困难, 了解学生输出准备的过程和结果, 审读 学生所做的 PPT。教师在提供帮助过程中要充分发挥其“脚 手架”作用, 即在备课时需要细化自己搭建脚手架的具体步 骤和内容, 以便自己监控与评估所提供帮助的有效性, 确 保学生的能力得到逐步提高。

4) 给予有针对性的反馈。目前教学中普遍存在的问题 是, 教师对学生完成的口头表达任务评价空泛, 对听众的 表现置之不理; 对学生完成的笔头表达任务过多注意语言 形式上的简单错误, 忽视语义表达的准确性和内容组织的 逻辑性。导致上述问题的原因很多。以口头表达任务评价 问题为例, 其产生的主要原因是教师事先对学生所要口头 报告的内容不知晓, 缺少事前准备, 自然临场难以对报告 的学生做出恰当反馈, 也无法控制听众的行为。因此, 学 生进行口头报告之前, 教师一定要了解口头报告的内容。 即使师生不能在课前进行面对面的交流, 教师也应该要求 学生将口头报告的内容提前发送到教师邮箱, 以便充分考 虑如何给予恰当的评价, 并事先设计任务确保听众的有效 参与。

\section{2 .2 学生任务}

1）尝试完成输出任务。这里强调的是输出任务的尝试 性, 学生出色完成任务不是此时应追求的目标。如果学生 能够说出自己在输出过程中的困难所在, 列出自己语言知 识的不足之处, 他们的任务就算尝试成功。

2) 学习和寻找新语言材料。学生要根据输出任务的要 求, 从提供的材料中尽快找到解决问题的答案。如果所提 供的新材料还不足以解决所有问题, 他们应该将问题记录 下来。解决问题的方案有两个: 一个是向教师讨教; 另一个 是自己查询图书资料, 搜索网络信息, 或向其他同学寻求 帮助。

3) 准备语言的产出活动。任务一旦需要两人以上参与, 就要配备协调人。协调人负责整个输出任务的分工和监控, 确保工作量均匀分配和任务顺利开展。口头表达任务的准 备工作大致分 3 步进行: (1) 组织内容; (2) 制作 PPT; (3) 预演。笔头表达任务的准备工作则大致包括: (1) 谋篇布局; (2) 撰写初稿; (3) 反复修改。 
4) 展示与欣赏输出成果。学生既要展示自己的输出成 果, 又要学习其他同学的输出成果。如果准备工作做得充 分, 展示时报告人、听众和教师各有贡献, 最终就能形成 合力，共同提高展示效果。

\section{WebQuest 与“输出驱动假设”的相似点}

\section{1 学习的主题类似}

WebQuest 的主题是 “一个需要解决的问题或者一个需 要完成的项目”, 这里的“问题或项目”即现实生活中的真实 任务, 所以这和“输出驱动教学”通过完成具有真实性的交 际任务 (即在日常生活中确实有可能面对的任务) 促进语 言学习是完全一致的。

\section{2 学习的宗旨及目标相同}

WebQuest 要求学生通过对网上信息资源的分析、综合 形成所面临问题或项目 (即真实任务)的解决方案, 并通过自 主探究或小组合作方式加以实施。而 “输出驱动教学” 要求 学生在围绕主题进行研究的过程中, 主动地获取知识, 并 要应用所学知识去解决选定的真实问题。显然, 这两种模 式的宗旨都是要通过研究解决实际问题来学习, 并且 学习所追求的最终目标也是一样的——不仅要能理解所学 的知识, 还要能够运用所学的知识去解决实际问题。

\section{3 实施流程相似}

不论是哪种 WebQuest 模式, 都包括“任务、过程、资 源、评价、总结”这几个基本步骤。其“选择一个能促进高 级认知发展的任务”与“输出驱动教学”中的“设计真实、符 合学生语言水平的输出任务”一致; 其“资源”对应“输出驱 动教学”中的“提供恰当的新输入材料”; 其“过程”相当于 “输出驱动教学”中的教师“提供适当的输出帮助”, 同时学 生“准备语言的产出活动”; 其“评价”对应“输出驱动教学” 中的教师“给予有针对性的反馈” 和学生 “展示与欣赏输出 成果”。

\section{4 评价体系一致}

两者都采用集“诊断性评价、形成性评价和总结性评 价”为一体的评价体系。该评价体系既关注学生学习的水 平, 也关注他们在学习活动中所表现出来的情感与态度, 以及合作交流能力和探究创新能力; 不仅有教师对学生的 评价, 还有学生的自我评价和相互评价。

\section{WebQuest 与“输出驱动假设”的完美结合}

5.1 “输出驱动假设”在教学实践中缺少为学生展示成果的 平台, 而WebQuest 恰好提供了这样一个平台。

学生肯定希望自己经过精心准备所完成的成果能有展 示的机会, 同时也希望看到别人的成果以取长补短。只有 为每个学生提供展示成果的机会才能激发学生的积极性, 提高教学效果。如果课上为每个学生提供展示成果的机会, 就要占用很多的时间。但大学英语每学期只有 64 学时, 这 就意味着一学期只能进行数量很少的活动。而 WebQuest 的“电子学习档案袋”恰好可以解决课时有限这一困难。“电 子学习档案袋” 是借助计算机技术来生成、存储、展示和分 析评价学生成果的档案资料, 反映学生的努力程度、进展 状况和成就水平。这些学习记录按照一定的顺序形成文档, 用于学习者对学习的回顾和自我评价。

5.2 “输出驱动假设”的有效开展离不开多元化的评估方式, 但多元化的评估方式耗时费力, 而 WebQuest 提供的评 价方法恰好可以解决这一问题。

为了让学生知道自己的作品有什么优势和不足, 可以 要求每位学生为所有的作品打分。如果想得到准确有效的 评价, 就必须具体规定各分量的评分等级。量规的使用能 大大降低评价的主观随意性。如果事先公布量规, 还可以 对学习者的作业起到导向作用。但汇总每个学生给其他作 品打出的分数这一工作需要消耗教师大量的时间和精力。

而 WebQuest 的作品分析法恰好解决了这一问题。

教师想了解调查学生对某个知识点掌握的自信程度、 比较学生当前能力水平与教学目标的差距、评价学生的学 习效果、对协作小组的贡献等可以采用调查问卷的方法。 但汇总调查问卷的结果又是一项耗时费力的大工程。而 WebQuest 的调查问卷法恰好解决了这一问题。

\subsection{WebQuest 系统有序, 有针对性。}

由于 WebQuest 的教学设计流程模板与“输出驱动假 设”的实施流程一致, 所以教师可以很方便地利用 WebQuest 这一平台为学生描述输出任务, 提供输入材料, 提供输出帮助, 给予有针对性的反馈。教师不用再借助 QQ 完成这些任务, 因为 WebQuest 比 QQ 更加系统, 一目了然, 有针对性。

\section{参考文献(References)}

[1] Norman, D., Gentner, D., \& Stevens, A. (1976). Comments on learning schemata and memory representation. In D. Klahr (Ed.), 
Cognition and instruction. Hillsdale, NJ: Erlbaum. H. Simpson, Dumb Robots, 3rd ed., Springfield: UOS Press, 2004, pp.6-9.

[2] Ogle, D. M. (1986). K-W-L: A teaching model that develops active reading of expository text. The Reading Teacher, 39 (6), 564-570. B. Simpson, et al, "Title of paper goes here if known," unpublished.

[3] Perkins, D. N., \& Simmons, R. (1988). Patterns of misunderstanding: An integrative model of misconceptions in science, mathematics, and programming. Review of Educational Research, 58(3), 303-326.

[4] Posner, G. J., Strike, K. A., Hewson, P. W., \& Gertzog, W. A.
(1982). Accommodation of a scientific conception: Toward a theory of conceptual change. Science Education, 66(2), 211-227.

[5] Strike, K., \& Posner, G. (1985). A conceptual change view of learning and understanding. In L. H. T. West \& A. L. Pines (Eds.), Cognitive structure and conceptual change. New York: Academic Press.

[6] Van Zee, \& Minstrell, J. (1997). Using questioning to guide student thinking. The Journal of the Learning Sciences, 6 (2), 227-269.

[7] Qiufang Wen. The application of the output-driven hypothesis to college English teaching. Foreign Language World, 2013, 6 